DOSSIER

\title{
LES FORMES MODERNES DE L'EMPRISE MANAGERIALE
}

\author{
DANIÈLE LINHART ${ }^{1}$ (D)
}

RESUME: Le management moderne se caractérise par ses dimensions contradictoires. Il prétend mettre au cœur de sa politique la mobilisation de l'intelligence, de la réactivité des salariés et il les entrave avec des dispositifs très contraignants de procédures et de reporting tout en attaquant leur expérience et leurs savoirs par une politique du changement permanent. C'est qu'il recherche avant tout à asseoir sa domination. Mais certains patrons cherchent d'autres modes de domination et pour ce faire « libèrent » leur entreprise...

Mots-clés: Management moderne. Changement perpétuel. Disqualification de la professionnalité. Logique taylorienne. Subordination.

\section{THE MODERN FORMS OF BUSINESS MANAGEMENT}

ABSTRACT: Modern Management can be characterized by its contradictory dimensions. It claims to the mobilization of intelligence and responsiveness of their employees at the center of their policy, as it impedes them with very restrictive procedures and reporting, while attacking their experience and knowledge through a policy of permanent change. It seeks, above all, to ensure one's dominance. Some employers, however, are looking for new ways of managerial control and, to do so, have decided to "set free" their business...

Keywords: Modern management. Permanent change. Disqualification from professionalism. Taylorian logic. Subordination.

\section{AS FORMAS MODERNAS DA GESTÃO EMPRESARIAL}

RESUMO: A gestão moderna é caracterizada por suas dimensóes contraditórias. Ela alega colocar no centro de sua política a mobilização da inteligência, a capacidade de resposta dos seus funcionários e coloca-lhes entraves com procedimentos e relatórios muito restritivos, ao mesmo tempo em que ataca sua experiência e seus conhecimentos por meio de uma política de mudança permanente. É que ela procura, acima de tudo, estabelecer seu domínio. Mas alguns patróes buscam outros modos de dominação e, para isso, "libertam" sua empresa...

Palavras-chave: Gestão moderna. Mudança permanente. Desqualificação do profissionalismo. Lógica tayloriana. Subordinação.

${ }^{1}$ Laboratoire GTM-CRESPPA (Genre Travail Mobilité - Centre de Recherches en Sociologie et Politique de Paris), Paris, France. E-mail: daniele.linhart@cnrs.fr

DOI: 10.1590/ES0101-73302019219374

Educ. Soc., Campinas, v.40, e0219374, 2019 


\section{Introduction}

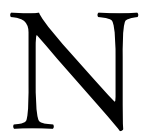

ombre de jeunes actifs reculent à l'idée d'intégrer le salariat et s'essayent à d'autres formes de mise au travail. Ils s'orientent vers l'auto entrepreneuriat, le travail dit indépendant, souvent ubérisé en lien avec des plateformes numériques; ils cherchent à créer leur propre entreprise, leur start up, ou cumulent en tant que slashers plusieurs petits boulots.

C'est que le monde du travail salarié peut avoir quelque chose d'effrayant ; il est de plus en plus associé à la souffrance, au mal-être, aux risques psychosociaux, aux addictions à des produits licites ou illicites (pour résister au travail) et pire encore aux suicides. Les media ainsi que des romans, films ou pièces de théâtre relaient dramatiquement cette réalité douloureuse.

Pourtant, les mêmes media, ainsi que de nombreux colloques, séminaires, salons d'expositions ou différents types « d'événements » mettent, par ailleurs, en scène une autre image du travail et des entreprises : on entend parler des "Directions des Ressources Humaines (DRH) du Bonheur et de la Bienveillance, des chief happiness officers, de séances de massage, de méditation, d'entretiens téléphoniques gratuits avec des psychanalystes, de salles de détente, d'open space accueillant, de télé travail pour limiter les déplacements etc... On y entend des discours selon lesquels les directions se disent préoccupées de faire " grandir » leurs salariés, affirment l'importance de tenir compte de leurs aspirations personnelles, et de reconnaitre la qualité de leur engagement au travail. La "vraie vie " serait au sein de ces entreprises où les directions misent sur les qualités humaines de leurs salariés, et affichent une rupture totale avec le management taylorien. Les nouveaux outils numériques le permettent, les nouvelles règles de la compétition internationale l'imposent : il faut mettre en valeur les capacités de réactivité, d'adaptation, d'intuition, bref la subjectivité des hommes et des femmes.

Comment expliquer cette vision contrastée du monde du travail où les DRH en appellent au bonheur alors que des salariés s'enfoncent dans le mal-être et la souffrance?

\section{La profonde ambiguïté du modèle managérial moderne}

Une partie de l'explication réside sans doute dans les contradictions inhérentes à ce projet managérial moderne, qui confrontent les salariés à des situations délétères et souvent inextricables.

D’un côté, on a vu se développer depuis les années 80 une politique systématique d'individualisation, de personnalisation, voire même de psychologisation et narcissisation de la relation des salariés à leur travail (DE GAULE- 
JAC, 2005). Tout d'abord une individualisation des objectifs et évaluations de leur travail, assortie de primes et salaires personnalisés qui instaurent une mise en concurrence entre eux. Puis une psychologisation et narcissisation de la relation au travail qui induit une mise en concurrence de chacun avec soi-même : on demande aux salariés de viser l'excellence, de se dépasser, de sortir de leur "zone de confort ", de relever les défis, d'étonner leur hiérarchie, et de se surprendre euxmêmes en mobilisant des potentialités qu'ils n'imaginaient même pas. Il y a, en ce sens, comme une surhumanisation des modalités de mise au travail car ce sont leurs qualités les plus personnelles, les plus intimes qui sont mises au cœur de la mobilisation des salariés et de leur évaluation. Désormais, la hiérarchie manage les émotions des salariés et gère leurs affects.

D'un autre côté, les directions ont imposé à ces mêmes salariés de travailler selon des prescriptions impératives, comme en témoigne l'important corpus de méthodologies, process, procédures, protocoles, bonnes pratiques benchmarkés, mis au point par les consultants des grands cabinets d'experts internationaux, à distance du travail concret, et destinés à encadrer le travail (DUJARIER, 2016). Car il ne s'agit pas, du point de vue du management, de s'en remettre à la professionnalité de leurs salariés. Son objectif reste toujours le même : ne dépendre en aucune façon des salariés, trouver impérativement les moyens de les obliger à renoncer à leurs valeurs professionnelles, citoyennes et morales pour n'endosser que celles de leur direction. L'enjeu est de parvenir à contraindre les salariés de travailler en fonction des seuls critères de productivité, qualité, rentabilité choisis par leur employeur ; il s'agit donc de créer les conditions d'une véritable subordination des travailleurs, une subordination effective et efficace, dans la continuité de la démarche taylorienne.

C'est Taylor, en effet, qui, à la fin du $19^{\text {ème }}$ siècle, a rendu la subordination des ouvriers possible en attaquant de front leurs métiers. Il avait compris que le savoir c'est du pouvoir et qu'il ne fallait pas le laisser aux ouvriers. Le modèle qu'il conçoit vise à déposséder les ouvriers de leurs savoirs, de leur expérience pour les transférer aux employeurs. Ceux-ci, avec leurs ingénieurs, vont se les approprier pour mettre en place une organisation du travail prescrite en tâches élémentaires répétitives assorties de modes opératoires très détaillés. A partir de ce moment, les ouvriers seront tenus de se conformer strictement à ce qui sera prescrit et tout manquement sera sanctionné. La contrainte et le contrôle sont désormais intégrés dans l'organisation du travail à laquelle les ouvriers sont soumis.

Auparavant seuls détenteurs des métiers et donc du savoir de la production, les ouvriers de métiers organisaient librement leur travail. Ils étaient en mesure alors d'imposer leur vision du travail et faire valoir leurs intérêts. En l'occurrence, ils veillaient à ne pas risquer des accidents ou une usure prématurée de leur force de travail et limitaient leur rythme de travail qu'ils alignaient par ailleurs sur les tarifs auxquels ils étaient payés. Ils pratiquaient ce que Taylor (1956) appelait « la flânerie 
systématique » et qui aboutissait à une faible productivité, dommageable, selon lui, pour le patron mais également pour les citoyens américains qui méritaient de disposer de produits de consommation plus nombreux et moins couteux. Son objectif était donc de trouver les conditions permettant à l'employeur d'imposer aux ouvriers d'autres méthodes de travail conçues pour atteindre une productivité maximale. Des méthodes de travail qui passaient par une attaque en règle des métiers ouvriers et l'invention d'une organisation du travail coercitive relayant les seuls critères d'efficacité et rentabilité décidés par l'employeur.

Dans la réalité des faits, le modèle ne s'est jamais appliqué comme tel. Face à une organisation nourrie de prescriptions pensées abstraitement par des ingénieurs à distance des aléas et des incidents multiples qui scandent toute activité de travail, les ouvriers ont développé des savoir, des savoir faire, une expérience adaptés aux contraintes locales de leur travail et qu'ils mettaient en ouvre collectivement, clandestinement, en outrepassant les consignes hiérarchiques et les modes opératoires imposés. Ils prenaient des risques mais permettaient au travail de se dérouler dans des conditions satisfaisantes, en combattant les dysfonctionnements de cette organisation taylorienne qui se prétendait scientifique et rationnelle. Ils la rendaient possible et efficace et permettaient ainsi à une organisation conçue sur l'éradication des savoirs et compétences ouvrières de fonctionner grâce aux savoirs et compétences informels, clandestins qu'ils reconstituaient sur le terrain. Tout ce que les sociologues, ergonomes et psychologues du travail ont étudié sous le concept de «travail réel » par opposition au travail formel prescrit. Les travailleurs vivaient subjectivement ce "travail réel " le plus souvent comme une réappropriation, certes parcellaire, mais néanmoins réelle, du sens du travail. Cependant, ils renforçaient le modèle bien plus qu'ils ne le combattaient.

Ce mode de mise au travail a trouvé ses limites sociales à la fin des années soixante du siècle dernier. Une révolte ouvrière s'est élevée dans nombre de pays industriels et particulièrement en France (trois semaines de grève générale avec occupation d'usines). L'urgence pousse alors le patronat à inventer un autre management capable de "réconcilier " les salariés et leurs employeurs. Celui-ci se fondera, pour inverser un rapport devenu trop défavorable aux employeurs, sur une individualisation des travailleurs, (comme on l'a évoqué), au prétexte de satisfaire leur besoin d'autonomie, de dignité et de reconnaissance. Les employeurs réhabilitent la subjectivité des salariés (elle est désormais présentée comme indispensable pour la performance des entreprises et le bien-être des travailleurs), mais dans la réalité ils cherchent à organiser une mise sous tutelle de cette subjectivité, sur le plan idéologique (par une psychologisation et narcissisation de la relation des salariés à leur travail) et organisationnel (à travers le déni de leur professionnalité par un corpus de procédures imposées).

L'individualisation va de pair avec une orientation qui cherche l'adhésion des salariés à la cause de leur direction, qui vise à arracher leur consentement 
afin qu'ils utilisent les marges d'autonomie, d'inventivité et créativité pour faire, en réalité, l'usage d'eux-mêmes le plus efficace et le plus rentable, du seul point de vue des critères décidés par la direction, critères qui sont restés dans la majorité des cas très tayloriens, c'est à dire focalisés sur l'économie des temps et des coûts. Chaque salarié doit ainsi se transformer en petit bureau des temps et des méthodes pour veiller à trouver en permanence des solutions d'organisation de son travail qui lui permettent de répondre à ces injonctions, chacun doit mobiliser ses capacités cognitives, émotionnelles, affectives pour atteindre ces objectifs inscrits dans la philosophie taylorienne rebaptisée. Il sera d'ailleurs évalué et jugé à l'aune de ses capacités personnelles pour y parvenir.

Ce qui est demandé aux salariés par le management moderne est de se mobiliser entièrement pour mettre en œuvre des méthodes de travail et des objectifs déterminés en dehors d'eux par des experts, des consultants indifférents le plus souvent aux spécificités du métier. C’est pourquoi des sociologues britanniques et français ont parlé d'autonomie contrainte ou contrôlée. Certes, le management requiert de ses salariés de l'inventivité et de l'intuition mais pour rendre efficaces et opérationnels des outils, des dispositifs décidés par d'autres et ailleurs, en fonction des seules logiques de rentabilité. Et, comme le remarque fort bien Diet (2013), il est explicitement demandé à chaque salarié de mettre intelligemment en œuvre des protocoles et des méthodologies dont l'existence même est un déni de son intelligence puisqu'ils sont là pour se substituer à son expérience et sa professionnalité. L'évaluation omniprésente des salariés qui détermine leur carrière dans l'entreprise, leur rémunération, se fonde sur cette contribution d'un type bien particulier. Et c'est leur personnalité tout autant que leurs compétences qui est passée au crible. Ce qui est demandé c'est une adhésion sur la base d'un enrôlement. Il s'agit d'accepter de prendre des risques, de se mettre en question, en danger, de sortir de "sa zone de confort ". Ne proposait-on pas, dans certaines entreprises, au cours des années 90 , aux salariés de sauter à l'élastique ou en parachute, de courir le marathon, pour apprendre à dominer leur peur, à dépasser leur limites, à chercher au fond d'eux-mêmes les ressources les plus personnelles pour faire face à des situations extrêmes ?

Le management moderne recherche et récompense, chez le salarié, essentiellement sa capacité d'adaptation, sa compréhension de ce que l'on attend de lui et son inventivité pour ce faire.

\section{Une « nouvelle » organisation du travail mais de vieux principes tayloriens}

En ce sens point de rupture avec le taylorisme. On se situe dans une réelle continuité avec l'option de base taylorienne qui consiste à récuser l'expérience et la professionnalité des salariés et de confier à des experts éloignés du ter- 
rain la responsabilité de définir les manières de s'y prendre au travail. Le taylorisme n'a pas disparu, son esprit est bien présent, la différence réside dans le fait qu'il ne s'impose plus autoritairement et quasi mécaniquement aux salariés mais qu'il suppose leur complicité active, leur adhésion. Les salariés sont censés devenir les relais convaincus, actifs et efficaces de ces méthodes tayloriennes.

Reste cette question fondamentale. Comment les directions font-elles pour arracher ce consentement ? La question est d'autant plus importante que les directions, notamment en France, sortaient d'une période d'intense contestation du taylorisme où se manifestait clairement la volonté des salariés de peser sur les orientations du travail. Certes, il y avait eu cette stratégie du cheval de Troyes consistant à individualiser et personnaliser la relation au travail pour désamorcer la capacité collective de contestation. Mais dès lors qu'une contribution active et inventive des salariés à la mise en œuvre des dispositifs inspirés de la logique taylorienne est requise, il faut plus. Il faut que les salariés adhèrent ou soient obligés d'adhérer.

Les porteurs de la modernisation du management français l'ont bien compris. Séduire, faire adhérer, cela fait partie des objectifs qui ont orchestré différentes phases de la modernisation du management français pour convaincre les salariés d'accepter de s'imposer à eux-mêmes la violence organisationnelle inspirée du taylorisme.

On distingue plusieurs phases qui représentent autant de tentatives de la part des employeurs d'opérer une métamorphose identitaire (LINHART, 1991) de leurs salariés pour les mettre en conformité avec les exigences du nouveau modèle qui se met progressivement en place.

Dans les années 1980, les entreprises françaises, notamment les plus grandes d'entre elles, privées comme publiques, développent de multiples dispositifs participatifs. La période s'y prête, les socialistes viennent d'arriver au pouvoir, et les lois Auroux en 1982 octroient de nouveaux droits aux travailleurs, notamment celui d'une expression directe et collective. Le patronat estime assez rapidement qu'il peut être intéressant de mettre la hiérarchie en dialogue avec des salariés réunis au sein de divers groupes, afin de faire passer des messages, de diffuser ses valeurs. L'idée d'instaurer une pacification interne via le dialogue et les échanges s'ancre dans l'esprit des responsables d'entreprise. L'enjeu est clairement d'amener les salariés à renoncer à leurs propres valeurs professionnelles et citoyennes (autour de questions aussi fondamentales que le travail bien fait, le travail utile, la possibilité de travailler selon les règles du métier) et d'adhérer, de façon consensuelle, à celles mises en œuvre par leur direction.

Cette démarche participative vise ainsi à aligner les points de vue des différents protagonistes sur les critères et les valeurs de l'entreprise. Groupes d'expression, cercles de qualité, et divers groupes ad hoc donneront l'occasion aux salariés de se retrouver, en présence de leur supérieur hiérarchique, pendant les 
heures de travail autour d'une table pour échanger autour de questions du travail et surtout entendre le point de vue du management.

Dans la foulée, les grandes entreprises s'affirmeront aussi en tant qu'institutions productrices de valeurs et d'éthique. Elles se lanceront au cours des années 90 dans l'élaboration et la diffusion de chartes éthiques, de codes déontologiques, de règles de vie, définissant les bons comportements professionnels, les manières adaptées de s'impliquer au travail et de tisser des relations avec les collègues, la hiérarchie, les clients. Anne Salmon (2000) parle d'offre éthique des entreprises, car celles-ci inventent une nouvelle éthique définissant ce qu'est un salarié vertueux : un salarié qui ne pense pas à ses intérêts égoïstes mais prend en compte ceux de son entreprise censée rassembler le bien commun. Ce salarié se doit de s'engager à fond, de viser l'excellence en permanence, de donner le meilleur de lui-même.

Le travail devient ainsi de plus en plus une affaire personnelle qui s'inscrit dans les valeurs de l'entreprise, à distance des enjeux plus citoyens. Les salariés sont systématiquement mis en concurrence les uns avec les autres mais également chacun avec lui-même puisqu'il doit sans cesse se dépasser.

Mais, en attendant que les choses murissent, que les générations évoluent, il faut parer au plus pressé. Prendre des mesures plus immédiatement efficaces qui permettront de mobiliser de façon rentable des salariés pas encore tout à fait convaincus, ni totalement séduits. Pour faciliter et accélérer la diffusion de l'idéologie et de l'éthique managériale, pour obliger les salariés à s'en remettre, sans plus attendre, aux seuls critères d'efficacité, de qualité et rentabilité voulus, l'entreprise moderne déploie ce que l'on pourrait appeler une politique de dissuasion des salariés, visant à désamorcer toute velléité de leur part de se prévaloir de leur métier, de leurs connaissances et expérience professionnelles pour imposer leur point de vue et leurs valeurs dans la réalisation de leur travail. Et pour ce faire, il s'agit, dans le plus strict respect de la philosophie taylorienne, de déposséder les salariés de leurs ressources professionnelles pour asseoir une domination assurant la prévalence des seuls critères choisis par les directions.

Comment peut on attaquer la professionnalité des salariés ? Comment peut on les déposséder de leurs savoirs, de leur expérience pour les maintenir dans un état de réelle dépendance et subordination?

\section{Une précarisation subjective des salariés}

Un processus de précarisation subjective prend alors la relève pour assurer le " consentement » des salariés et atteindre le même effet que celui produit par la précarisation objective (Linhart, 2015). 
La précarité objective est un moyen coercitif efficace, on le sait. Ceux qui sont en CDD, intérim, temps partiel imposé, contrat saisonnier, stage, ne sont enclins ni à critiquer, ni à chercher à imposer leurs aspirations, valeurs et convictions. Ils espèrent, en majorité, une titularisation, une stabilisation dans l'emploi. Ils comprennent vite qu'il vaut mieux se conformer strictement à ce que l'on attend d'eux, apprendre même à devancer les attentes de leur hiérarchie. La population précaire est par essence plus facile à manœuvrer, contraindre et convaincre. Mais si le nombre de salariés précaires augmente régulièrement, $80 \%$ des salariés sont en emploi stable ou bénéficient d'un statut de fonctionnaire. Il est moins aisé d'exercer sur eux l'emprise qui les obligera à appliquer les procédures, les bonnes pratiques décidées pour eux.

La précarisation subjective, au moyen de la politique du changement permanent, aboutit à déstabiliser les salariés de sorte qu'ils se sentent eux aussi sur le fil du rasoir, dans un sentiment d'insécurité et de vulnérabilité et n'aient d'autre choix que de se rabattre sur les procédures, les méthodes standard, comme sur des bouées de sauvetage.

Le changement permanent est d'ailleurs présenté dans la rhétorique comme une vertu en soi, comme un signe de progrès par opposition aux routines, rigidités, pesanteurs. Il est également présenté comme une nécessité de s'ajuster aux évolutions de plus en plus fréquentes du marché, des technologies et des mentalités. Mais il faut remarquer que nombre d'entre elles, sont artificielles, en ce sens qu'elles ne sont pas dictées uniquement par cette accélération du temps mais qu'elles sont la conséquence d'autres logiques.

Parmi celles-ci, on peut signaler les pratiques des managers qui soumis au diktat de la mobilité cherchent à marquer leur passage d'une réforme qui manifestera leur inventivité, leur détermination, leur audace, leur aptitude à prendre des risques. Quand on sait que la durée de leur passage avoisine deux ou trois ans et que chaque nouvelle arrivée entraine de nouveaux bouleversements, on mesure l'impact que ces pratiques peuvent avoir sur les personnes encadrées. C'est une dynamique qui s'auto alimente sans répit.

Le phénomène du recours très systématique à des cabinets de consultants, des Sociétés de Services en Ingénierie et Informatique implique des stratégies commerciales qui vont dans le même sens de l'accélération temporelle. Pour fidéliser leurs clients et les inciter à renouveler leurs commandes, les consultants organisent l'obsolescence de leurs dispositifs et orchestrent une succession de modes organisationnelles. La pratique du benchmarking (BRUNO; DIDIER, 2013) va dans ce sens qui incite à une veille permanente pour repérer dans les autres entreprises, des pratiques efficaces et les introduire chez soi. Cela induit une propension constante à la modification, au changement. 
Mais il y a des pratiques de changement motivées par la volonté de rendre la subordination effective, c'est à dire par la volonté d'asseoir une autorité sans faille sur les salariés. Il s'agit dès lors d'un changement permanent qui vise à rendre inopérants les connaissances, les savoirs, l'expérience accumulés par les salariés. Restructurations sans fin de services et départements, recompositions incessantes de métiers, changements accélérés de logiciels, mobilités systématiques imposées, externalisation d'activités suivies de ré internalisations, déménagements multipliés, tout cela a pour fonction de rendre les connaissances des salariés, leurs savoirs, et expérience sans utilité.

C’est la professionnalité des salariés qui est ainsi attaquée comme leurs collectifs d'ailleurs (dans la continuité de l'individualisation menée systématiquement comme on a pu le voir), soit deux composantes fondamentales de la capacité des travailleurs à faire face aux difficultés et éviter d'être submergés, à titre personnel, par les tensions, contradictions et sentiments d'injustice au travail. En réalité, ce qui protège les individus au travail, c'est avant tout leur professionnalité qui leur permet de mettre une distance entre ce qu'ils font et ce qu'ils sont, à l'aide de règles de métier, de normes de travail auxquels ils adhèrent, qu'ils partagent avec leurs collègues, et qui font sens pour eux. Ces règles, ces normes, ces pratiques partagées et validées avec d'autres auxquels ils s'identifient, font rempart contre les difficultés, le mal-être et les angoisses qu'elles peuvent susciter.

Le nouveau modèle innove donc mais dans la continuité avec l'ancien modèle taylorien. S'il réintroduit la subjectivité bannie par Taylor, il persiste à dévaloriser la professionnalité des salariés d'une manière certes différente mais tout aussi efficace: celle qui consiste à rendre obsolètes les compétences et l'expérience professionnelle des salariés par une politique de changement perpétuel.

\section{L'entreprise en burn out}

Le management participe ainsi d'une casse des savoirs, des compétences, de l'expérience des salariés. Ceux-ci ne sont pas réduits à l'état d'exécutants sans aucune initiative comme ont pu l'être les ouvriers du temps de Taylor, mais ils se trouvent ravalés au rang d'apprentis permanents, d'apprentis à vie. Dans ce contexte où tout change tout le temps, ils perdent leurs repères, ne peuvent bénéficier de leur expérience, ni de celle leurs collègues ou de leur hiérarchie (qui bouge tout le temps). Ils ne sont plus en mesure de maitriser cognitivement leur travail. Ils doivent sans cesse développer des efforts démesurés pour essayer de reconquérir des repères, le recul nécessaire pour développer leur activité, tout en sachant que très vite, tout cela volera en éclat sous les coups d'une énième réforme, d'un énième changement. Cela évoque les travaux de Sisyphe. Les salariés sont plongés dans une sorte d'état de précarité subjective. Ils seront alors plus enclins à se conformer aux consignes, prescriptions et ordres hiérarchiques qu’à contester l'organisation imposée de leur travail. 
Le burn out, qui affecte de plus en plus de salariés, découle de cette logique, qui exige de chaque salarié des efforts démesurés et continus pour se maintenir au niveau et continuer à atteindre les objectifs. Il découle aussi de la disqualification que subissent les salariés attaqués dans leur image de soi, dans leur valeur. Les salariés ne peuvent plus se faire confiance à eux-mêmes, car ils n'ont plus les ressources qui leur permettent d'être de bons professionnels.

On pourrait logiquement en déduire que c'est l'entreprise qui est elle même en burn out, qu'elle se consume intérieurement. En détruisant les savoirs de leurs salariés, en anesthésiant leur inventivité, leur créativité, leur imagination, leur gout de l'expérimentation, leur sens du travail bien fait et leur valeurs citoyennes, les directions dévorent les capacités de performance de l'entreprise.

S'il faut prendre au sérieux les discours managériaux et politiques sur la nécessaire performance des entreprises, il faut aussi prendre au sérieux les ravages que cause le modèle managérial moderne. Dans la continuité du taylorisme, il écrase la professionnalité des salariés qui constitue le socle de l'entreprise, il bride leurs initiatives, les enferme dans une impuissance généralisée. Il limite la mise en œuvre du «travail réel », celui qui est adapté aux contraintes locales et fluctuantes du travail. Il compromet ainsi la capacité de l'entreprise à innover et trouver des solutions pour assurer sa pérennité. Le développement du numérique, le recours à l'intelligence artificielle amplifient cette orientation.

Une critique managériale de ce management moderne émerge depuis plusieurs années. Une sorte de consensus se dégage de nombre d'ouvrages récents publiés par des gourous, consultants ou dirigeants d'entreprises pour critiquer les travers du management à l'œuvre dans la plupart des entreprises.

Ce courant déplore des contraintes inutiles : trop de procédures imposées, trop de contrôles sous forme de traçabilité et de reporting. Cela va à l'encontre du bien-être des salariés qui se trouvent assiégés, étouffés. Le management moderne contrarie, selon ce courant, leurs aspirations à plus d'autonomie, de possibilités de se réaliser dans leur travail, de s'y reconnaître. Et si les salariés vont mal, la performance des entreprises en est nécessairement pénalisée.

Cette critique portée par des managers met en évidence les aspects contreproductifs et délétères du management moderne qui mènent au burn out comme au bore out, aux risques psychosociaux, au mal-être, à la souffrance et aux suicides au travail. Mais à y regarder de près, on prend conscience que derrière cette critique, et prenant appuie sur elle, se profilent des « solutions " qui risquent d'aggraver encore plus la situation des travailleurs sur le plan du travail comme de l'emploi.

C'est à ce virage préoccupant que notre société est d'ores et déjà confrontée. Nous sommes à un véritable tournant : la critique du management est forte, elle est largement partagée par différentes parties prenantes de cette société, mais les stratégies déjà élaborées et travaillées idéologiquement construisent un monde 
du travail plus inquiétant encore, alors que peinent à émerger, du côté des travailleurs, des contre modèles convaincants.

\section{Entreprises « libérées » par leur patron : vers la création d'un nouveau salariat de confiance?}

Il existe, en France désormais des « entreprises libérées » par leur patron (on peut lire les livres de " patrons libérateurs » comme Alexandre Girard (2017) ou Jean-François Zobrist (2018) et elles ont fait des émules : les entreprises « en voie de libération " (AirBus, Décathlon, Kiabi, MAIF), les entreprises " responsabilisantes " (entreprise Michelin), " concertatives ", (entreprise Hervé), " horizontales ", à " pyramide inversée " ... Il s'agit de grandes entreprises, de Petites et Moyennes Entreprises ou de Mutuelles, voire de Ministère.

Lidée de base de ces " patrons libérateurs » est qu'il faut faire confiance aux salariés pour qu'ils déploient le travail réel efficace, nécessaire et ajusté. Ce qui importe, pour ces managers critiques, c'est de permettre aux travailleurs de gérer, réguler eux-mêmes leur travail, pour s'y sentir à l'aise et y être plus efficaces. Ils veulent faire toute sa place au travail réel car il est adapté aux situations de travail qui sont de plus en plus fluctuantes et imprévisibles. Ils veulent miser sur la fluidité, l'agilité, ne pas laisser les prescriptions rigidifier les capacités d'adaptation et la réactivité.

Ils veulent aussi que les salariés de base prennent en charge d'autres missions auparavant réservées à l'encadrement intermédiaire, ou aux fonctions supports.

Déployer un travail réel qui mobilise au mieux les savoirs et l'expérience, rien de vraiment nouveau: c'est l'approche Toyotiste, avec le lean management, censée être au cœur du management moderne mais qui n’a jamais été appliqué selon les tenants de la libération des entreprises. Comme l'explique le gourou de ce mouvement des " entreprises libérées ", Isaac Getz avec Brian Carney (2013) : on propose aux salariés de Toyota des manières efficaces de faire leur travail mais on ne les oblige pas à continuer à les appliquer s'ils découvrent une meilleure méthode. Et au lieu d'employer les instruments de contrôle habituels, Toyota fournit à ses salariés des instruments d'auto contrôle. C'est une différence de taille avec ce que vit la main d'œuvre dans la plupart des grandes entreprises" (GETZ; CARNEY, 2013, p. 51).

On a là le postulat de la libération des entreprises : les salariés savent mieux faire sur le terrain que leur hiérarchie et leur direction. Ces libérateurs préconisent donc le principe de subsidiarité: les manières de travailler doivent se penser localement, face aux impératifs et aléas du travail et non en fonction de règles décidées hors sol. Les règles dans le management moderne «ne se bornent pas à saper le moral des salariés; elles empêchent la grande majorité d'entre eux de faire ce qui conviendrait » écrivent encore Getz et Carney (2013, p. 20). 
Leur cible est donc en permanence le moral de leurs salariés pour que ceux-ci se mobilisent de façon performante, mais et c'est une restriction non négligeable, dans le cadre des orientations définies unilatéralement par leur dirigeant, leur « leader " qui est porteur d'une "vision ». Cette " vision » définit des objectifs pour l'entreprise en termes de positionnement sur le marché, d'efficacité et de rentabilité, en termes d'organisation du travail, de répartition des responsabilités et des objectifs à atteindre selon les métiers et les secteurs. Les salariés doivent s'imprégner de cette "vision ", l'intérioriser et la mettre en œuvre, dans leur travail réel, du mieux qu'ils peuvent, en bénéficiant d'une certaine dose d'autonomie concédée, qui lorsqu'elle n'est pas toujours concrètement possible et réelle doit leur paraître pourtant toujours présente.

Car l'impression d'autonomie est, selon ces " patrons libérateurs " toute aussi importante que sa réalité pour le moral des salariés et l'efficacité de leur travail. Comme l'expliquent encore Getz et Carney (2013, p. 68) :

Quand un individu a l'impression de jouir d'un degré de contrôle important sur un événement ou une situation, il le juge moins stressant et peut même y voir un défi. [...] Par exemple, face à un afflux soudain de clients, une vendeuse qui a l'impression de bien contrôler son travail aura confiance en elle; elle se dira qu'elle va trouver le moyen de s'adapter et de gérer cette charge supplémentaire. De sorte que ses émotions loin de devenir négatives, peuvent même lui inspirer une impression positive de défi à relever.

Les salariés doivent croire en leur leader libérateur, en sa «vision», ils doivent se constituer en followers. A noter que la liberté donnée aux salariés, désormais considérés comme des égaux, est déclarée dans certains domaines et pas d'autres, selon les entreprises. Ainsi, «l'ouverture est [...] la règle chez Richards Group à une exception près : la rémunération. Dans cette agence, discuter de sa paie avec ses collègues est un motif de licenciement" (GETZ, CARNEY, 2013, p. 68).

Si les salariés libérés intègrent bien la «vision », on les organisera de façon à ce qu'ils puissent s'auto manager, s'auto motiver, s'auto diriger, s'auto contrôler et s'auto discipliner. Ils le feront individuellement et au sein d'équipes pluridisciplinaires qui seront le cour de l'entreprise. Ces équipes qui choisissent leur animateur parmi eux, auront à assumer, en complément de leur tâche propre, des missions qui relèvent des Ressources Humaines, du domaine commercial voire financier.

Cela permet à la direction de "l'entreprise libérée » de réaliser d'importantes économies financières car elle se passera de nombreux cadres, notamment des cadres de proximité et cadres intermédiaires ainsi que de certaines fonctions supports, sans pour autant tomber dans l'anarchie.

La vertu, sinon l'astuce de ce modèle est de transformer l'ensemble des salariés en salariat de confiance. Le salariat de confiance était auparavant, notamment 
dans la période des Trente Glorieuses, (les trente années suivant la deuxième guerre mondiale) représenté en France par la catégorie peu fournie des cadres. Jusque dans les années 1960, les cadres relevaient d'un rapport de confiance avec leur direction comme le développe Paul Bouffartigues (2001). Ce rapport spécifique se caractérisait par la confiance manifestée par la direction à ses cadres à travers la délégation d'une parcelle de son pouvoir et de son d'autorité. Et en retour par la confiance sous forme de loyauté que lui assuraient ces mêmes cadres. Au cœur de cette relation, la promesse d'une carrière sous forme d'un parcours promotionnel assuré.

Depuis les années 80 , le nombre des cadres ne cesse de progresser de façon spectaculaire, en même temps que se dégradent leurs conditions et le contenu de leur travail. Ils sont de plus en plus soumis aux contraintes de procédures, protocoles et reporting, aux dispositifs de fixation d'objectifs unilatéralement imposés et d'évaluations hiérarchiques. Leurs carrières, de fait, sont plus limitées. Leur loyauté se redéfinit alors : elle s'adresse moins à leur direction, à leur entreprise qu’à leurs propres valeurs, à leurs propres intérêts professionnels et personnels.

Le courant des " entreprises libérées " opère ainsi un déplacement : elle se détourne d'une partie des cadres notamment intermédiaires (qu'elles éliminent) pour tenter de retrouver avec tous les salariés une relation de confiance basée sur une délégation de parcelles d'autonomie en contrepartie d'une loyauté totale.

Si le bénéfice pour la direction est facile à identifier, quel est celui des salariés qui se voient confier des charges supplémentaires ? Il est, proclament les «patrons libérateurs ", du côté du bien-être, de la qualité du moral, de l'esprit d'équipe, du sentiment de liberté et d'autonomie, du plaisir de s'identifier à une "vision " et un leader, de la possibilité d'adapter des règles et des procédures pour mieux faire son travail. Mais il ne se traduit qu'exceptionnellement sur le plan des rémunérations, celles ci n'augmentent pas toujours et si elles augmentent ce n'est que de montants relativement faibles. Par ailleurs, la marge d'autonomie des salariés reste le plus souvent très cadrée, ils doivent se fixer des objectifs qui sont en résonnance avec ce que la direction attend d'eux, travailler de la façon qui est escomptée par leur hiérarchie, et qu'ils intègrent au fur et à mesure de leur « libération ». Leur charge de travail augmente et ils ont à régler des problèmes autrefois réservés à l'encadrement, notamment de médiations de contradictions qui se manifestent dans tout travail au sein d'une l'équipe. Ils ont à gérer toutes les tensions liées à la gestion des augmentations de salaires, des primes, de l'absentéisme, des relations avec les clients... Les travaux sociologiques qui portent sur l'encadrement de proximité montrent bien l'ampleur, la complexité de ces tâches et la charge mentale qu'elles représentent sur le plan cognitif comme sur celui émotionnel.

Leur charge de travail se complexifie, s'alourdit mais les récompenses sont à chercher du seul coté symbolique, celui de faire partie d'une "entreprise libérée " où le dirigeant fait confiance aux salariés, et mise sur leurs capacités à se faire les relais loyaux, efficaces, fiables et disponibles de sa vision de leader. Celui-ci 
n'hésitera pas à manifester tous les signes de sa confiance. Ainsi Alexandre Girard (2017), patron de Chronoflex a pu réaliser son rêve, partir pour un tour du monde en voilier pendant un an avec sa famille et retrouver son entreprise florissante...

Le plus important dans cette libération réside dans la force de l'intériorisation, par les salariés, de la «vision» de leur leader. Ceux qui n’adhèrent pas sont poussés à partir, mais les salariés qui acceptent les nouvelles règles du jeu doivent travailler en permanence en fonction de cette «vision». Ils doivent renoncer de fait à leurs propres valeurs professionnelles ou citoyennes, à leurs propres intérêts au prix d'une charge de travail plus exigeante, plus complexe et parfois déstabilisante. Les salariés acceptent de se mettre sous tutelle.

\section{Améliorer le salariat, supprimer le lien de subordination}

Les nouvelles orientations des managers critiques du management moderne ont de quoi inquiéter.

Il y a d'un côté une propension, on l'a évoqué au début, à miser sur une mobilisation de travailleurs hors salariat. Il s'agit de toutes les formes d'externalisation qui aboutissent à un travail considéré comme indépendant mais en réalité largement sous tutelle économique et organisationnelle.

Il y a une propension d'un autre côté à transformer les entreprises en véritables sectes tombant sous la houlette du leader et imposant un tri entre ceux qui y croient et ceux qui n'y croient pas, imposant surtout à ceux qui y croient de renoncer à la critique.

Dans les deux cas, ce sont les modalités de la subordination qui sont renouvelées. La subordination est désormais associée au consentement explicite des travailleurs. Dans un cas, ils sont considérés comme libres parce que dotés d'un statut d'emploi indépendant, alors qu'ils sont soumis en réalité au diktat de la plateforme numérique qui cadre leur travail. Dans l'autre, ils sont présentés par leur leader comme des égaux, qui partagent sa «vision» alors qu'ils n'ont pas d'autre choix que de s'y conformer strictement et de l'alimenter par leur travail. Et l'on peut faire l'hypothèse que c'est cela l'objectif premier des nouvelles stratégies de mise au travail : trouver les moyens de déléguer aux travailleurs la responsabilité de leur propre subordination.

Or c'est le principe même de la subordination qui pose problème et qui est à l'origine de bien des dimensions délétères et contre performantes du management. C'est cette subordination qui mine les possibilités du salariat de se transformer en un modèle qui prenne en compte les différents enjeux sociaux, économiques et écologiques du travail.

Car d'un côté, ce principe de subordination inscrit dans le contrat salarial pousse certains à rechercher des formes substitutives d'emploi indépendant, et de 
l'autre, il peut conduire à un management plus dévastateur encore dans de nouvelles versions comme celle de "l'entreprise libérée ", deux options fort différentes mais qui aboutissent à rendre plus difficile encore toute mise en débat de la finalité du travail.

Si le salariat doit être critiqué dans sa forme actuelle responsable de lourdes souffrances au travail et de risques psychosociaux, il présente cependant de sérieux avantages. Ces avantages sont à relier à sa dimension collective qui a débouché sur des droits, des garanties et protections arrachés de haute lutte par les travailleurs et leurs organisations représentatives au cours de l'histoire. Ils sont à relier à l'héritage d'un destin commun face à des difficultés vécus collectivement, même si les politiques managériales ont cherché à instaurer une individualisation et personnalisation de la relation de chacun à son travail.

Il y a ainsi dans ce salariat des éléments favorables à la mise en débat collective des enjeux fondamentaux du travail, sa finalité sociale et économique (les conditions, le contenu et le sens du travail, la prise en compte des besoins travailleurs mais aussi des consommateurs ou usagers, la répartition des richesses) comme ses dimensions écologiques.

Mais cette capacité de mise en débat est compromise par cette dimension totalement archaïque du salariat, que représente le lien de subordination inscrit dans le contrat de travail et qui paralyse et entrave les salariés. C'est ce lien de subordination qui rend possible les dérives managériales et organisationnelles et fige l'entreprise dans ses dimensions les plus irrationnelles et injustes.

Un salariat sans subordination est possible et envisageable, qui préserverait les capacités de protection et les droits des salariés, si on arrivait à penser autrement l'entreprise. La vraie modernisation serait de repenser le salariat à travers l'invention d'une entreprise capable de prendre en compte les véritables enjeux que véhicule le travail. Et de ne pas laisser le champ libre aux seuls dirigeants des entreprises qui renouvellent sans cesse les modalités d'appropriation du travail, dans une dialectique (Taylorisme/post taylorisme/«entreprise libérée») infernale.

\section{Références}

BOUFFARTIGUES, P. Les cadres : fin d'une figure sociale. Paris: Ed la Dispute, 2001. BRUNO, I.; DIDIER, E. Benchmarking: l'Etat sous pression statistique. Paris: Ed Zones, 2013.

DIET, E. Changement catastrophique, acédie et résistance au...changement . Revue de Psychothérapie psychanalytique de groupe, n. 61, n. 2, p. 19-40, 2013. http://dx.doi. org/10.3917/rppg.061.0019

DUJARIER, M. A. Le management désincarné; Enquête sur les nouveaux cadres du travail. Paris: La Découverte, 2016.

GAULEJAC, V. La société malade de la gestion. Paris: Le Seuil, 2005. 
GETZ, I.; CARNEY, B. Liberté et Cie. Quand la liberté des salariés fait le bonheur des entreprises. Paris: Fayard, 2013.

GIRARD, A. Le patron qui ne voulait plus être chef. Paris: Flammarion, 2017.

LINHART, D. La comédie humaine du travail; de la déshumanisation taylorienne à la sur humanisation managériale. Toulouse: Erès, 2015.

LINHART, D. Le torticolis de l'autruche; l'éternelle modernisation des entreprises françaises. Paris: Le Seuil, 1991.

SALMON, A. L'offre éthique des entreprises, une production de l'ordre économiques? Paris : Editions du CNRS, 2000.

TAYLOR, F. W. La direction scientifique des entreprises. Paris: Dunod, 1956.

ZOBRIST, J. F. La belle histoire de Favi; l'entreprise qui croit que l'homme est bon. Raleigh USA: Ed Lulu, 2018.

\section{À propos de l'auteur}

Danièle Linhart et docteur en sociologie. Directrice de recherche émérite au Centre national de la recherche scientifique (CNRS). Membre de l'équipe GTM (Genre travail Mobilités) du Laboratoire CRESPPA (centre de recherches sociologiques et politiques de Paris), France.

Reçu le ler février 2019.

Accepté le 9 septembre 2019. 